\title{
Are people happy when they smile? \\ Affective assessments based on automatic smile genuineness identification
}

\author{
Monica PERUSQUIA-HERNANDEZ \\ NTT Communication Science Laboratories \\ perusquia@ieee.org
}

\begin{abstract}
Smiles are one of the most ubiquitous facial expressions. They are often interpreted as a signalling cue of positive emotion. However, as any other facial expression, smiles can also be voluntarily fabricated, masked or inhibited with different communication goals. This review discusses automatic identification of smile genuineness. First, emotions and their bodily manifestation are introduced. Second, an overview of the literature on different types of smiles is provided. Afterwards, different techniques used to investigate smile production are described. These techniques range from human video-coding, bio-signal inspection, and novel sensors that, together with automated techniques using machine learning, aim to investigate facial expression characteristic's beyond human perception. Next, a general summary on the spatiotemporal shape of a smile is provided. Finally, remaining challenges regarding individual and cultural differences are discussed.
\end{abstract}

Keywords: Affective Computing; smile genuineness; facial expressions of emotion; sensing technology. 


\section{Embodied Emotions}

Emotions usually have a bodily manifestation which can be measured. These embodied changes that occur when we experience an emotion include facial movement, body movement, temperature, and skin conductivity changes. Since emotions are private states, embodied cues have been used as an alternative to measuring emotion (Oberman, Winkielman, \& Ramachandran, 2007; Wilson, 2002). Early theories of emotion suggest that changes in our body state are not only a symptom of experiencing an emotion, they also claim that our body state is the reason for the subjective feeling of an emotion (Darwin, 1872; James, 1884). More recent theories suggest that emotion can also be triggered endogenously by cognitively appraising a certain situation (Scherer, Schorr, \& Johnstone, 2001).

Facial expressions are one of the embodied cues of emotion. The Basic Emotion Theory (BET) proposed prototypical facial expressions believed to be universal and mapped to a specific felt emotion (Ekman, 1992, 1999; Galati, Scherer, \& Ricci-Bitti, 1997). However, humans excel at voluntarily controlling their facial expressions. Facial expressions are often fabricated to provide misleading information about the producer's emotional state. They can also be voluntarily used as a social signalling tool with several functions, even in the absence of felt emotion. Thus, it is hard to assume there is an emotion whenever we observe a facial expression; even though BET suggests that a facial expression occurs whenever there is an emotion (Ekman, 2003; Ekman \& Friesen, 1969a, 1982a; Ekman \& Rosenberg, 2005).

The lack of coherence between facial expressions and emotion is a challenge for affective assessments. Facial expressions of emotion have often been regarded as two extremes of a spectrum (Figure 1). On one hand, we have spontaneous facial expressions that are involuntary reflexes arising from an experienced emotion. These facial expressions co-occurring with emotion are often called spontaneous or genuine (Guo, Zhang, Liang, \& Yan, 2018). On the other hand, there are completely voluntarily produced facial expressions that are emitted with a social signalling goal. These are often referred-to as posed, deliberate, or voluntary (McGettigan et al., 2015). 


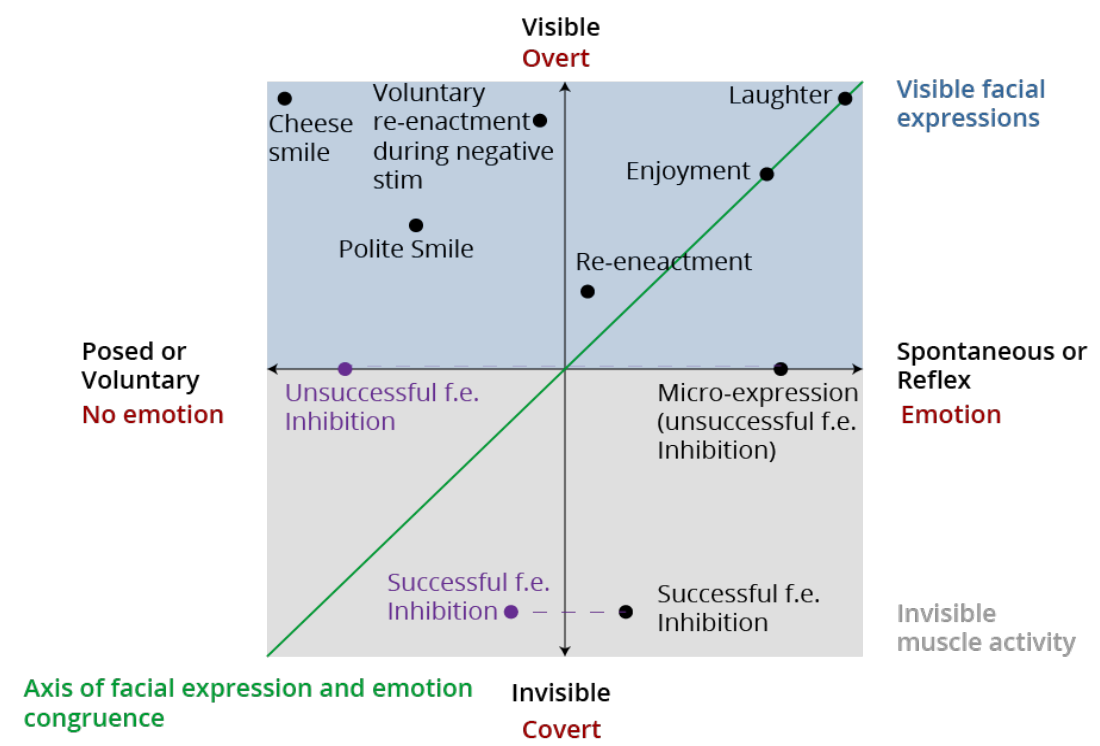

Figure 1. The facial expression space along the genuineness and visibility axes. The BET suggests that spontaneous smiles co-occur with emotion. However, they can also be posed in the absence of it. These are two extremes of a continuum. On the other hand, some smiles are overtly displayed, while others are very subtle or actively inhibited. If we could assume congruence between emotion and facial expression, all smiles would fall on the green axis. Nevertheless, smiles of different kinds can be placed outside it. For example, a cheese smile posed for the camera is often displayed without feeling a particular emotion. Moreover, micro-expressions are expressions caused by a strong emotion but voluntarily suppressed. When leaked, they are in the boundary of visibility, but there could also be cases where facial inhibition is successful. Even when a poker-face is achieved, covert bodily cues such as EMG, or EDA activity can be measured.

The degree of genuineness of a facial expression of emotion is easier to study as categorical variable. However, as posed and spontaneous expressions often appear mixed with each other, more and more researchers are considering the importance of analysing them in a continuous manner. For example, people can voluntarily produce smiles that are spontaneous when thinking of past events or making an association with something funny (i.e., reenactment). We also encounter situations where we exaggerate our expression of emotion to make sure others understand how we feel. Thus, we should not only care about the ground truth of the facial expression production, but also, about how people interpret certain facial expressions (Dawel et al., 2017). It has also been argued that the message transmitted by each facial expression includes both biologically basic and socially specific messages depending on whether we look at the beginning or at the end of each facial expression (Jack, Garrod, \& Schyns, 2014).

The interpretation of facial expressions of emotion by observers has led to many insights on how we perceive other's emotions. This is the core of the behavioural ecology 
view (BECV) of facial displays. In BECV, a facial expression of emotion is interpreted in terms of how observers understand it, and not in terms of the inner affective state that caused it (Crivelli \& Fridlund, 2018). As with the genuineness spectrum, other models fall in between the BET and the BECV extremes. The Componential Processing Model (CPM) of emotion appraisal (Mehu \& Scherer, 2012) suggests that both internal and social cues affect how people respond. This can be further understood by looking at components of the facial expression. For example, some Facial Action Units (AU) described by the Facial Action Coding System (FACS) (Ekman \& Friesen, 1982b) are reliable and others versatile. Reliable AUs seem more difficult to control than versatile AUs. Furthermore, reliable AUs' activation has a stronger effect on the meaning of the perceived facial expression (Mehu, Mortillaro, Bänziger, \& Scherer, 2012).

Given their social relevance, controlling our facial expressions is desirable independently of whether we are experiencing a strong emotion or not. The degree of control over our facial expressions has been especially relevant when studying micro-expressions. These are expressions leaked despite efforts to either deliberately or unconsciously conceal an emotion. To achieve concealment, there are several strategies: (1) simulation or deliberately displaying an emotion; (2) masking of the facial expression with another expression or speech; and (3) inhibition of the facial expression (Ekman \& Friesen, 1975). The inhibition strategy has often been used to experimentally elicit micro-expressions in the so-called neutralising paradigm (Yan, Qi, Jing, Chen, \& Fu, 2013). Since inhibition is a technique to control our own facial expressions, we could map them in a spectrum ranging from overt to covert (Figure 1). Overt facial expressions are expressions displayed for everyone to see. On the other hand, covert facial expressions are those that are very subtle or suppressed to a point where they are visually almost unperceivable. Micro-expressions could be placed at the boundary of what is visually perceivable. Additionally, there are cases where an emotion is experienced without any visually perceivable facial expression, that nevertheless has an embodied manifestation that is measurable only with other sensing modalities. This is often the case for facial mimicry, which is so subtle that it is usually assessed with Electromyography (EMG).

All in all, both the production and the perception perspectives are important. We might ask whether people are happy when they smile, i.e., production perspective; or ask what 
makes people believe that someone else is happy, i.e., perception perspective. In this review, the focus is on the production of smiles, and the affective ground truth behind a smile. In particular, the goal is to summarise previous research aimed to answer the questions: (1) what are the characteristics of a spontaneous smile?; and (2) how does a spontaneous smile differ from a posed smile?

\section{Smiles and their multiple facets}

Smiles are the prototypical facial expression for happiness (Ekman \& Friesen, 1969b). They are identified by an upward lifting of the lip corners, caused by activation of the Zygomaticus Major muscle (Darwin, 1872). In the FACS, this movement is labelled as AU12. Although AU12 is the most characteristic feature of a smile, other facial movements often cooccur with it and are perceived as integral factors of these smiles. The Orbicularis Oculi (AU6, AU7), which raises the cheek and tightens the upper and lower eye lids, has also been described to be involved in felt smiles (Ekman \& Friesen, 1982a). This is the so-called Duchenne marker (Ekman, Friesen, \& Davidson, 1988), named after Duchenne (1862) who was the first to associate this movement with felt smiles during positive emotions.

Ekman, Friesen, and Davidson (1988) described different types of smiles. False smiles are deliberately produced to convince another person that a positive emotion is felt when this is not the case. These false smiles were further divided into phony smiles that appear in the complete absence of emotion; and masking smiles where a strong negative emotion is felt but the smile is an attempt to conceal those feelings by appearing positive. Interestingly, they also described miserable smiles, which occur when someone is distressed, and accurately convey the impression that the person is not happy. Ekman (1992) has described more than 15 smiles with different meanings and morphology. Indeed, smiles are not only an expression of joy. They have also been shown to denote reward, affiliation, and dominance. From these, reward smiles largely overlap with felt smiles, and are displayed during rewarding situations. Affiliation smiles are expressed to create social bonding. Finally, dominance smiles are displayed when negotiating a hierarchy to signal superiority (Rychlowska et al., 2017). These three smile types are considered to be part of a continuum, and thus combinations are possible. 
The perception of smiles depends on several factors such as context, order effects, observer's beliefs, status, and culture (Rychlowska, Manstead, \& van der Schalk, 2019).

Another smile taxonomy classifies smiles in four categories (Stewart, Bucy, \& Mehu, 2015). Enjoyment smiles which involve the zygomatic muscles pulling the lip corners up together with engagement of the eye muscles; yet the jaw is not dropped in amusement. Amusement smiles are usually accompanied by laughter and activity in the lower face such as jaw drop. Contempt smiles are characterised by unilateral lip corner pull and tightening of the lip corner (AU14). Posed smiles are equivalent to Ekman's false smiles. Finally, controlled smiles are an attempt to constrain the extent of amusement or self-satisfaction in response to external stimuli. At times, controlled smiles might also entail an exaggeration of felt amusement or a power display. In this sense, the controlling label seems to be a moderating factor between posing a smile and expressing it spontaneously.

At the micro-expression level, smiles are rare. A characterisation of the timing of microexpressions revealed that leaked happiness displays (AU12, AU12+AU6) were less frequent than those of other emotions (Yan et al., 2013). Even though amused facial displays were common, they were much slower than the 500 ms boundary for micro-expressions. A similar result was found when assessing subtle smiles with distal EMG (Perusquía-Hernández, Hirokawa, \& Suzuki, 2017b). This suggests that smiles are easy to control when they are overt, but they are harder to inhibit.

\section{Measuring smiles}

As noted by the aforementioned works, people are not always happy when they smile. Smiles are common, and they are one of the most versatile facial expressions. This has made them a popular target for measurement. Smile measurement, similarly to that of other facial expressions, has been prominently visual. From Duchenne's pictures to recent high-frame rate videos or even using infrared cameras. Nevertheless, there are also other measurement techniques such as EMG, which has been used to assess facial mimicry for decades.

In recent years, automatic identification of facial expressions has gained popularity. Its main advantage is its reliability, or the extent to which the results can be reproduced under the 
same conditions. Current automatic identification algorithms take only physical factors into consideration and do not mix them with prior personal beliefs. Moreover, most sensors have higher temporal resolution than self-report. Several reviews have been conducted to summarise the different signals that can be used for technology-afforded emotion recognition (Calvo \& Mello, 2010; Janssen et al., 2013; Vinciarelli, Pantic, \& Bourlard, 2009). However, most systems can only claim a high perceptual agreement between facial movement perceived by a machine and a human. This does not necessarily inform us about the underlying emotion because of the lack of congruence between facial expressions of emotion and felt affect. Therefore, accurate ground truth labels beyond AU coding are required for these systems to work, and more often than not, multiple sensing modalities are required to achieve accurate results hinting at the presence or absence of felt affect. There are different methods of establishing the ground truth used to train these systems. These methods include using the video-coding of facial expressions, self-reported labels, and labels related to the context in which the data was acquired. In the following sections, different methods to measure smiles and other (covert) co-occurring affective behaviour are described. When research is available, the focus is on distinguishing between posed and spontaneous smiles.

\section{Visually}

Smile genuineness has been assessed mainly through the morphology and dynamic features of the smile itself.

Human perception. Human video-coding of facial expressions is considered to be the ground truth for automatic identification systems. The FACS has been widely used to manually annotate facial expressions. FACS rating involves identifying Action Descriptors, consisting of muscular movements involving multiple muscles, on a frame-by-frame basis from their onset to offset. Peak contraction is then noted on a discrete scale. The identified AU configurations can then be analysed for co-occurrence of emotional states, and inferences can be made by experts in the frame of different theories. The main advantage of the FACS is that the observable AUs are described and counted before making more subjective inferences about the message they convey (Ekman, Friesen, \& Hager, 2002).

Most classic research on facial expressions is based on this coding system. The aforementioned research on smile categorisation usually indicates the differences in terms of 
AUs as identified from a static frame, as they often involve different facial muscles (Ekman \& Rosenberg, 2005). Arguably, the main difference is the activation of the Duchenne marker only during spontaneous smiles (Ekman, 1999; Ekman, Friesen, \& Davidson, 1988; Ekman, Friesen, \& O'Sullivan, 1988; Guo et al., 2018). However, other studies have found that this muscle is activated in both felt and non-felt smiles (Krumhuber \& Manstead, 2009; Namba, Makihara, Kabir, Miyatani, \& Nakao, 2016; Schmidt, Bhattacharya, \& Denlinger, 2009). The Duchenne marker might also signal smile intensity instead of smile authenticity (Girard et al., 2019; Krumhuber \& Manstead, 2009; Messinger, 2002) given that the ability to display this marker can be learned (Mai et al., 2011).

The dynamics of facial expressions have been deemed critical to infer their meaning. The information that can be obtained from static images is limited (Krumhuber \& Manstead, 2013; Namba, Kabir, Miyatani, \& Nakao, 2018; Orlowska, Krumhuber, Rychlowska, \& Szarota, 2018). However, the FACS can also be used to analyse temporal sequences of AUs. For example, the temporal sequence of spontaneous amusement shows an expression of AU6+AU12, followed by AU6+AU12+AU25. In other words, eye and lip movement preceded mouth opening. In contrast, posed amusement was a display of AU25 followed by AU6+AU12+AU25, indicating that mouth opening happened before lip and eye movements (Namba et al., 2016). Moreover, spontaneous smiles usually have apex coordination during which muscle contractions in different parts of the face peak at the same time (Ekman \& Rosenberg, 2005).

While the FACS assumes expert training in identification of individual AUs, human distinction of posed and spontaneous smiles has also been studied from the perspective of naive participants. Several studies have found that laypersons have only a moderate ability to distinguish between these two types of expressions (Bartlett, Littlewort, Frank, \& Lee, 2014; Zloteanu, Krumhuber, \& Richardson, 2018). Human judges can distinguish between posed and spontaneous displays of emotion with moderate accuracy for amusement, surprise, disgust, and sadness. However, this sensitivity depends on the dynamic presentation of the facial display (Namba et al., 2018). Furthermore, only moderate accuracy was achieved by human judges when distinguishing between acted reward, affiliative, and dominance smiles with a 
consistency of approximately $65 \%$. Nevertheless, the prototypical characteristics of each smile type might have been enhanced by the actors (Orlowska et al., 2018).

Despite its informative nature, video-coding has several limitations. First, it is time consuming, and often requires multiple trained coders to reach high agreement levels. Second, video-coding is only suitable to perceptually label facial movement. Inferences of a person's intention or lack of intention by distinguishing between posed and spontaneous smiles are inaccurate. The agreement between video-coders decreases considerably when making inferences about the nature of the smiles being coded. Moreover, laypersons' accuracy on inferring whether a smile is spontaneous or faked is modest (57\% on average) independent of their ethnic match to the producer of the smile. Third, participants are biased to believe that smiles were posed even if there was an equal number of posed and spontaneous smiles (Perusquía-Hernández, Ayabe-Kanamura, \& Suzuki, 2019a).

Computer vision. Computer Vision (CV) has been widely used to automatically identify facial expressions or posture (Bettadapura, 2012; Guo et al., 2018). State-of-the-art cameras can measure a couple of hundreds of frames per second, which has enabled detection of fast facial expressions even at the micro-expression level (Oh, See, LeNgo, Phan, \& Baskaran, 2018).

Automatic feature tracking was used to describe the timing of motion in posed and spontaneous smiles of young adults of Euro- and African-American background (Cohn \& Schmidt, 2004). The duration of the onset phase of posed smiles is comparable with spontaneous smiles. However, spontaneous smiles have smaller amplitude and a longer, more consistent relation between amplitude and duration. A linear discriminant classifier using timing and amplitude measures of smile onsets achieved a 93\% recognition rate, and timing measures alone led to $89 \%$ recognition rate. Therefore, dynamic and morphological features enable automatic facial expression analysis to discriminate among the message values of morphologically similar expressions.

By using a combination of human video-coding and the Automated Facial Image Analysis (AFIA), deliberate smiles have been found to have larger onset and offset speeds, amplitude of movement and offset duration. In addition, deliberate smiles show no difference 
in asymmetry compared to spontaneous smiles (Schmidt, Ambadar, Cohn, \& Reed, 2006). In another study, human video-coding and AFIA revealed higher asymmetry of posed smiles, with higher onset amplitude, and longer duration than spontaneous smiles (Schmidt et al., 2009).

Another study explored spontaneous face-to-face interactions of people being amused or polite to an interlocutor. After participants were asked to label their own facial expressions, dynamics of the pre-labelled smiles were explored using the Sophisticated Highspeed Object Recognition Engine (SHORE) software. The findings confirmed that amused smiles tend to have longer duration. Moreover, amused smiles had more symmetry in the velocities of the rise and decay than polite smiles. Finally, the fastest decay was found in shared polite smiles (Hoque, Morency, \& Picard, 2011).

The Extended DISFA is a FACS annotated video dataset of posed and spontaneous facial expressions. The authors introduced a peak-valley temporal characteristics estimation where multiple peaks can occur within one facial expression (Mavadati, Sanger, Mahoor, \& Street, 2016). Since then, several similar datasets have become available. The UvA-NEMO database contains 1240 videos of spontaneous and posed smiles (Dibeklioglu, Salah, \& Gevers, 2015). Its bench-mark reached an identification accuracy of close to $90 \%$. Recently, a number of works have tried to distinguish posed from spontaneous smiles. The use of spatial patterns has been shown to achieve about $90 \%$ accuracy in this task (Wang, Wu, \& Ji, 2016). State-of-theart methods reach an identification accuracy of up to $92.90 \%$ by lip and eye landmark movements tailored to different age groups (Dibeklioglu et al., 2015). Other algorithms using spatiotemporal features, identified by restricted Boltzmann machines, have been able to achieve up to $97.34 \%$ accuracy with the UvA-NEMO database, and $86.32 \%$ with the Spontaneous vs. Posed Facial Expression (SPOS) database (Yang \& Wang, 2017). Finally, using the OpenFace software to extract facial landmarks from the UvA-NEMO database, genuine smiles have been shown to have higher values for onset, offset, apex, and total duration, as well as offset displacement, and a variable termed Irregularity-b (the $S D$ of the apex phase) than do posed smiles (Guo et al., 2018).

One of the main limitations of camera based techniques is their lack of robustness in noncanonical situations. These include occlusion, high movement, and lighting changes. Most of the existing datasets for facial expressions are captured within the visible light (VIS) spectrum. 
However, light can change with time and location, causing significant variations in appearance and texture. Another CV-based alternative is to measure facial expressions from near-infrared (NIR) video sequences. NIR imaging combined with LBP-TOP (Local Binary Patterns from three orthogonal planes) features provides an illumination invariant description of face video sequences. Component-based facial features on the Oulu-CASIA NIR\&VIS facial expression database, a support vector machine (SVM) and sparse representation classifiers led to detection of smiles with up to $83.75 \%$ of accuracy. The results were also robust against illumination variations (Zhao, Huang, Taini, Li, \& Pietikäinen, 2011).

Head tilting. Besides facial movement, head movements such as tilting have been shown to convey several emotions. A bowed head is perceived as sad and submissive. It is usually displayed during inferiority emotions (i.e., shame, embarrassment, guilt, humiliation, and respect). Conversely, a raised head is perceived as more dominant and displaying greater superiority emotions because of the greater apparent size that it creates (i.e., contempt and pride). This movement is even described in common language phrases such as "to turn up one's nose at someone”. Moreover, a raised head also connotes happiness (Mignault \& Chaudhuri, 2003).

Head movement using a cylindrical head model can be calculated from CV-based measurements to later compute the direction of correlation among smiles as determined from the AU12. Lip-corner displacement and head pitch were negatively correlated as predicted for smiles of embarrassment, while a positive correlation may be typical of smiles of enjoyment (Cohn et al., 2004). Furthermore, head and shoulder movement features added useful information to facial features when distinguishing posed from spontaneous smiles (Valstar, Gunes, \& Pantic, 2007). Finally, head movement as measured from an Inertial Measurement Unit (IMU) attached to the head explained best the differences between posed and spontaneous smiles in a predictive model. Participants moved more during spontaneous smiles. In contrast, they moved more in between posed smiles. These head movements were rather revealing and led to recognition rates above $95 \%$ of accuracy (Perusquía-Hernández, Ayabe-Kanamura, \& Suzuki, 2019b). 
Thermal imaging. Thermal imaging can be used to discriminate AUs from specific facial temperature patterns in Regions of Interest (ROI). The source of thermal changes on the face is related to both autonomic and voluntary changes. This includes cardiovascular responses, perspiratory responses, respiratory responses, and muscular responses (Cho \& Bianchi-Berthouze, 2019).

Similar to infrared imaging, thermal imaging aims to solve the problem of illumination that hinders CV performance with regular cameras. The fusion of vision-based and thermalbased imagery is a common strategy to fill the gaps where visual perception is limited, and to solve challenges for complex emotions (Nguyen, 2015). Infrared imaging aids the observation of the affective-state-specific facial thermal variations. Significant facial thermal feature points along major facial muscles were derived with pixel-grey level analyses. Using these, clusteranalytic classification of transient thermal features for spontaneous smile identification reached $87.5 \%$ of accuracy, and pretended smiles were classified with an accuracy of $62.5 \%$ (Khan, Ward, \& Ingleby, 2009). Furthermore, a spatial pattern approach on thermal imaging was used to classify nine different AUs and to differentiate their speed and strength of muscle contractions. Principal Component Analysis loadings were used to determine the latency between onset, apex and offset of a facial expression. The apex, or the temperature peak, was detected at $1282 \pm 439 \mathrm{~ms}$ after the end of the baseline for fast AUs, and at $1976 \pm 550 \mathrm{~ms}$ for slow AUs. Finally, using this technique AU6+AU12 were correctly identified in $15.2 \%$ of the cases using a K-nearest neighbour model (Jarlier et al., 2011). Furthermore, out of five facial sub-regions, temperature information in the forehead and the cheek region differ between posed and spontaneous expressions in their variance, their mean of the absolute values, and the variance of the absolute value of the temperature difference matrix of smiles (Wang et al., 2013).

Other studies have investigated facial skin temperature distribution on mixed thermal facial expressions of six basic expressions and 12 mixtures of those basic expressions. ROIs associated to mental stress were considered (i.e., periorbital, supraorbital and mouth). Vector mappings of the temperature variations led to groups of positive emotion induced facial features and negative emotion induced facial features. ROIs containing mixed expressions were 
less temperature inducing than corresponding basic expression's ROIs (Saha, Bhattacharjee, De, \& Nasipuri, 2016).

All in all, thermal imaging is a good alternative to enhance CV-based techniques beyond human visual perception by capitalising on the permanency of thermal Infrared (IR) signals. Although this method is still prone to occlusion and motion artefacts, it can detect facial expressions at the AU granularity without focusing on skin displacement. The occlusion problem is partially addressed by the use of portable thermographic systems embedded on mobile phones or helmets. These technologies might one day provide alternatives for worn sensors to detect physiological changes.

\section{Physiologically}

Besides visually perceivable (facial) behaviour, there are other measurable cues that can inform about the nature of a smile. These include Electromyography (EMG); changes in skin conductance also known as Galvanic Skin Responses (GSR) or Electrodermal Activity (EDA); and body thermodynamics. These measures have the potential to provide extra information to assess naturally occurring emotions where visual facial expression analysis might fail. For example, in uncontrolled, high movement environments; poker-face-like facial expression inhibition; and in blends of spontaneously and deliberately displayed smiles such as exaggerated smiles.

\section{Surface Electromyography}

EMG has been extensively used to study different facial expressions by placing recording electrodes near the muscles (Cacioppo \& Tassinary, 1990; Ekman \& Rosenberg, 2005; Oberman et al., 2007; Oberman, Winkielman, \& Ramachandran, 2009; Schmidt \& Cohn, 2001; Tassinary \& Cacioppo, 1992; van Boxtel, 2010), or with wearable devices that do not obstruct the face. These wearables have been used to detect the six basic emotions except happiness (Chen, Yang, \& Wang, 2015), and smiles from joint muscle activity (Gruebler \& Suzuki, 2014). Both its high temporal resolution and its close measurement of the body is advantageous for the EMG-based identification of subtle expressions that are visually imperceptible (Tassinary \& Cacioppo, 1992). Moreover, surface EMG has revealed that spontaneous smiles have 
different magnitudes, speeds, and duration than posed smiles (Cohn \& Schmidt, 2004; Schmidt et al., 2006).

\section{Distally measured Electromyography}

Posed and spontaneous smiles can also be distinguished by employing wearable facial distal EMG. In this method, facial movement remains unobstructed by placing the electrodes on the sides of the face, thus, increasing ecological validity. Distal EMG measurements are possible through volume conduction whereby the electrical activity generated by each muscle spreads to adjacent areas (van Boxtel, 2010). The information picked up by the electrodes is then used to approximate different sources of muscular activity using Blind Source Separation (BSS) methods such as Independent Component Analysis (ICA) (Comon, 1994). The resulting components are relevant for smile and frown detection as EMG measured from the Corrugator Supercilii and Zygomaticus Major muscles is robust and of sufficient magnitude, when compared to other facial muscles (van Boxtel, 2010). Thus, robust (subtle) smile detection even in presence of non-affective facial movements such as chewing gum and biting (Gruebler \& Suzuki, 2014; Oberman et al., 2007; Perusquía-Hernández et al., 2017b). This technique can also be used to distinguish posed from spontaneous smiles. Spatial and magnitude feature analysis provided an accuracy of about $74 \%$ in this task. Spatio-temporal feature usage increased the accuracy to around $90 \%$ and reduced the inter-individual variability. Therefore, both types of smiles differ as regards onset and offset times rather than magnitude (PerusquíaHernández, Hirokawa, \& Suzuki, 2017a). Furthermore, distal EMG decriptions showed that spontaneous smiles and smiles faking enjoyment differ in magnitude, onset time, and onset and offset speed independently of the producer's ethnicity (Perusquía-Hernández, AyabeKanamura, \& Suzuki, 2019a).

Posed and spontaneous smile detection using both $\mathrm{CV}$ and facial distal EMG methods were compared to investigate whether invisible potentials were informing the distinction. The mean accuracy of an intra-individual spatial features algorithm was $88 \%$ for $\mathrm{CV}$, and $99 \%$ for EMG. With intra-individual spatio-temporal features, the mean accuracy was $87 \%$ for $\mathrm{CV}$, and 91\% for EMG. This suggests that EMG probably has the advantage of being able to identify covert behaviour that cannot be detected visually in intra-individual models (PerusquíaHernández, Ayabe-Kanamura, Suzuki, \& Kumano, 2019). However, it is still a challenge to 
derive subject-independent models from EMG. Furthermore, there is a trade-off between not covering the face (distal measurement), and the precision with which fine-grained spatial movements such as AUs can be detected.

\section{Skin conductance}

Autonomic responses, such as Electrodermal Activity (EDA) or skin conductance, are expected to occur with spontaneous facial behaviour (Kreibig, 2010), as they happen without the interference of volition (Christopoulos, Uy, \& Yap, 2016). An increase of EDA is usually observed in emotions other than non-crying sadness, acute sadness, contentment, and relief (Kreibig, 2010). Additionally, negative emotions are usually associated with more prominent autonomic responses than positive emotions (Boucsein, 2012). Thus, EDA responses might differ depending on the experienced valence. Moreover, EDA is also suitable as a wearable affective assessment tool due to its ease of measurement (Girardi, Lanubile, \& Novielli, 2017; Silveira, Eriksson, Sheth, \& Sheppard, 2013). Previously, EDA was measured simultaneously from the hand and the neck of participants displaying spontaneous and posed smiles. Neck and hand activity was highly correlated, and EDA signals carry information about the spontaneity of the measured smiles, as shown by a classification accuracy of about 90\% (PerusquíaHernández, Ayabe-Kanamura, \& Suzuki, 2019b). Although EDA changes are a highly relevant feature that is less subject to volitional changes, it is prone to several artefacts and confounding factors such as environmental temperature changes, physical exertion, and sheer movement. Therefore, it needs to be interpreted carefully and, in most cases, combined with other sensing modalities.

\section{Novel sensors}

Recently, other sensors have been developed to detect facial expressions in a portable and private manner, even when there is high occlusion. This is particularly important for Virtual Reality applications; or for experiments where the cameras needs to be avoided to reduce behavioural changes due to the feeling of being observed.

Wearable photoelectronics. Photo-reflective sensors embedded with smart eyewear have been proposed as an alternative to measure facial expressions. A glass-type wearable equipped with 16 photo-reflective sensors can detect facial expressions by analysing the light 
reflection changes caused by skin deformations with a SVM classifier (Masai et al., 2017, 2015). Seven facial expressions, including smiles, can be recognised with an accuracy of up to $98.7 \%$. Despite working with light, the device is also robust to wearer's facial direction changes and slight changes in the position of the glasses. Moreover, $92.8 \%$ accuracy was achieved regardless of facial direction and removal-remount; 78.1\% accuracy for repeatability; and $87.7 \%$ accuracy in case of positional drift. Furthermore, when assessing spontaneous and posed smiles evoked with instructions, reflection intensity distribution and temporal features yielded a mean accuracy of $94.6 \%$ in user-dependent training (Saito, Masai, \& Sugimoto, 2020). This method derived similar temporal smile signatures as those observed from CV and EMG, whilst having the advantage of being robust for movement artefacts, and individual differences.

Acoustic sensing. Interferometry is a technique using skin-worn ultrasonic transducers to create acoustic interference patterns inside the wearer's body. These patterns are identifiable signatures as they are affected by the movements of the body. Interferi is an on-body gesture sensing technique using acoustic interferometry (Iravantchi, Zhang, Bernitsas, Goel, \& Harrison, 2019). When applied to the face, it can be used to detect posed smiles with $94.2 \%$ accuracy, and continuous tracking of smile intensity without exceeding $9.5 \%$ error; even when wearing a virtual reality headset. The potential to identify smiles when facial movements are restricted is its main advantage, but it is also its biggest weakness.

\section{The shape of a smile}

The aforementioned works hint that, independently of the measurement method, smiles have several common features. These features can be divided into spatial and temporal features. Spatial features are related to how the smile looks, and can be described with a single frameshot. Temporal features are related to how the smile characteristics change over time, and they can be described with plots depicting temporal changes.

Spatial features include mainly lip movement (AU12), eye movement (AU6, AU7), mouth opening (AU25), and their respective intensities. Moreover, depending on the type of smile, these can be accompanied by head rotations and occlusion caused by the hands. For example, smiles of embarrassment are often accompanied by covering the face with the hands 
or by leaning the head forward (Cohn et al., 2004); and dominant smiles might be accompanied by tilting the head backwards (Mignault \& Chaudhuri, 2003).

Temporal features are often described as magnitude and speed changes of a specific movement from a baseline (i.e., neutral face), to a maximum point, and returning to the resting position. To reiterate for emphasis, the start of the movement is often referred to as onset. The maximum magnitude of the change is referred to as apex. Finally, the end of the change is the so-called offset. This is the general smile envelope identified by CV, thermal, EMG, and optical sensors. However, it is only a simplified description. In reality, the shape of a smile is not only a triangular envelope. Some researchers consider more complex models like the Attack, Decay, Sustain, Release (ADSR) Model (Nakayama, Takano, Matsubara, Suzuki, \& Terasawa, 2017). This model assumes that after the initial peak of activity, there is a slight decrease followed by a sustain phase where the smile is held until it is finally "released" from sight (see Figure 2 for examples). Indeed, smiles tend to be a long-lasting facial expression, as evidenced by the infrequency of micro-smiles. However, the duration of the sustain phase is difficult to determine. Many smiles peak multiple times without fully returning to the baseline. This has led to several peak detection approaches described previously (Mavadati et al., 2016; Perusquía-Hernández et al., 2017a). Moreover, as a short smile composed from the first bout of laughter transforms in sustained laughter, one spatially identifiable smile unit is composed of many temporally-defined smile units which are more challenging to identify from a single feature type (Perusquía-Hernández et al., 2017b).

Despite the ambiguous definition of smile offsets and temporal units, it is possible to observe a familiar shape in several studies (Cohn \& Schmidt, 2004; Jarlier et al., 2011; Mavadati et al., 2016; Perusquía-Hernández, Ayabe-Kanamura, \& Suzuki, 2019a; PerusquíaHernández et al., 2017b; Saito et al., 2020; Schmidt et al., 2006). Moreover, different studies have shown that when posing a smile for the camera, or under instruction, the decay of the smile is sharper than that of spontaneous smiles of enjoyment (Perusquía-Hernández, AyabeKanamura, \& Suzuki, 2019a; Perusquía-Hernández et al., 2017b; Saito et al., 2020). This might be the most distinct feature of posed smiles elicited in this manner, and it seems easy to distinguish given the high accuracy of several detection systems. However, it can be argued whether this feature would extend to fake enjoyment smiles (Perusquía-Hernández, Ayabe- 
Kanamura, \& Suzuki, 2019a); or to smiles where there is a blend of genuineness and voluntary control such as the case of micro-expressions, and damped or controlled smiles.

A) Temporal envelope of a smile

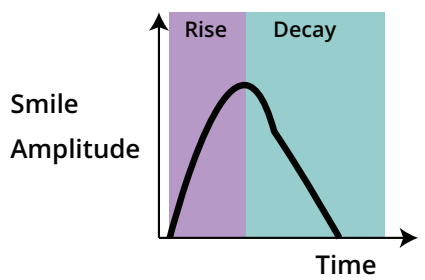

C) Example spontaneous smile

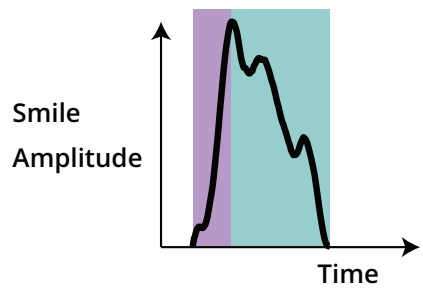

B) ADSR model

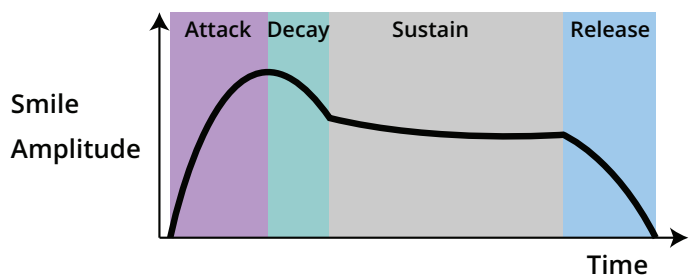

D) Example posed smile

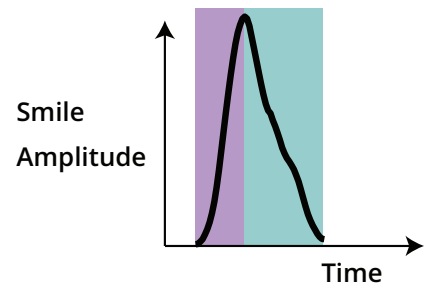

Figure 2. Examples of typical smile envelopes plotted from their temporal dynamics. A) The basic temporal envelope for an smile going from onset, reaching their maximum amplitude on the apex, and then decaying until the offset point. B) the Attack, Decay, Sustain, Release (ADSR) further identified a sustain phase where the smile is on hold. C) An example of spontaneous smile envelope with longer decay time and multiple peaks. D) An example of a posed smile faking enjoyment with only one peak.

\section{Methodological assumptions, unresolved issues, and open questions}

So far, automatic identification of facial expressions has proven useful to characterise the statistical distribution of both spatial and temporal features. Moreover, automatic identification has reached significantly high accuracy levels. Nevertheless, automatic identification is usually a black box tool that seldom allows us to explore the learned differences between different types of facial expressions. To fully take advantage of such tools, we should strive not only for high accuracy, but also to understand why a certain method is performing better than another. This is important in order to understand the psychological implications of newly developed techniques.

Moreover, we should also be careful about the paradigms used for data collection, as these might introduce biases and confounding factors in the developed algorithms. Despite the good results claimed in previous studies, the temporal dynamics of the reported posed smiles could have been affected by the duration of the instruction given to the participants. Therefore, 
further exploration is needed to confirm the differences between the temporal dynamics of several types of posed and spontaneous expressions.

Furthermore, it is important to note that most previous studies have used only Western, educated, industrialised, rich and democratic (WEIRD) samples for their studies despite evidence indicating that cultural diversity influences the production and perception of smiling behaviour (Henrich, Heine, \& Norenzayan, 2010). Smile and laughter displays by citizens of countries with ancestral diversity are easier to decode by observers, and they smile more in response to certain stimuli than residents of countries that lack ancestral diversity (Niedenthal, Rychlowska, Wood, \& Zhao, 2018). Additionally, a study using large-scale computer vision found that people from more individualist cultures display more brow furrowing, whereas smiling depends on both culture and setting. Participants from more individualist countries were more expressive in a facility setting than at home, while participants from more collectivist countries were more expressive at home. Male participants displayed less smiling and more brow furrowing than female participants, especially in individualist countries (McDuff, Girard, \& el Kaliouby, 2017). Moreover, posed or polite facial expressions might differ depending on cultural background (Thibault, Gosselin, Brunel, \& Hess, 2009; Thibault, Levesque, Gosselin, \& Hess, 2012) and racial match (Hourihan, Benjamin, \& Liu, 2012; Meissner \& Brigham, 2001). Only few studies targeted participants with mixed cultural and ethnic backgrounds (Cohn et al., 2004; Perusquía-Hernández, Ayabe-Kanamura, \& Suzuki, 2019a). Future research should embrace diversity, as this is likely to play a factor in the generalisation of automatic recognition.

\section{Concluding remarks}

The plethora of smile types, elicitation, and measurement techniques have made it difficult to reach a clear conclusion on the signatures of each smile type. Nevertheless, there are some commonalities found in most of the aforementioned studies. First, people are not always happy when they smile. There is still an open debate on whether smiling will eventually make people feel better due to the facial-feedback hypothesis (James, 1884). Even so, it is certain that at the moment of production, the source of the smile is not always enjoyment nor amusement. Smiling can also be faked or exaggerated with a social signalling goal, and the message transmitted might depend more on the perceptual context and the perceiver's beliefs 
than the producers' facial features alone. When considering smile production features, the Duchenne Marker has been believed to signal smile genuineness. However, there are several studies that cast doubt on this view, and provide evidence that it can be voluntarily produced. More recent studies also started to focus on the temporal dynamics of the smiles for both production and perception. These dynamics include phases that raise, sustain, and decay.

Finally, automatic smile identification is capable of augmenting our understanding of smile production beyond the limitations of human perception. These technologies will also become helpful to further assessments of the relationships among the expression, experience, and recognition of emotions. Understanding the relationships between these domains of emotion will help to shed light on the mechanisms of our affective experiences and the ways in which we infer their psychological significance.

\section{References}

Bartlett, M., Littlewort, G., Frank, M., \& Lee, K. (2014). Automatic Decoding of Facial Movements Reveals Deceptive Pain Expressions. Current Biology, 24(7), 738-743.

Bettadapura, V. (2012). Face Expression Recognition and Analysis: The State of the Art. Pre-Print. Retrieved from http://arxiv.org/abs/1203.6722

Boucsein, W. (2012). Electrodermal Activity. Boston, MA: Springer US.

Cacioppo, J., \& Tassinary, L. (1990). Inferring psychological significance from physiological signals. American Psychologist, 45(1), 16-28.

Calvo, R., \& Mello, S. (2010). Affect detection: an interdisciplinary review of models, methods, and their applications. IEEE Transactions on Affective Computing, 1(9), 18 37.

Chen, Y., Yang, Z., \& Wang, J. (2015). Eyebrow emotional expression recognition using surface EMG signals. Neurocomputing, 168, 871-879.

Cho, Y., \& Bianchi-Berthouze, N. (2019). Physiological and Affective Computing through Thermal Imaging: A Survey. Pre-Print. Retrieved from http://arxiv.org/abs/1908.10307

Christopoulos, G., Uy, M., \& Yap, W. (2016). The body and the brain: measuring skin conductance responses to understand the emotional experience. Organizational Research Methods, 22(1), 394-420. 
Cohn, J., Reed, L., Moriyama, T., Xiao, J., Schmidt, K., \& Ambadar, Z. (2004). Multimodal coordination of facial action, head rotation, and eye motion during spontaneous smiles. In Proceedings - sixth ieee international conference on automatic face and gesture recognition.

Cohn, J., \& Schmidt, K. (2004). The timing of facial motion in posed and spontaneous smiles. International Journal of Wavelets, Multiresolution and Information Processing, 2, $121-$ 132.

Comon, P. (1994). Independent component analysis, A new concept? Signal Processing, 36(36), 28-314.

Crivelli, C., \& Fridlund, A. (2018). Facial Displays Are Tools for Social Influence. Trends in Cognitive Sciences, 22(5), 388-399.

Darwin, C. (1872). The Expression of the Emotions in Man and Animals. New York D. Appleton and Company.

Dawel, A., Wright, L., Irons, J., Dumbleton, R., Palermo, R., O’Kearney, R., \& McKone, E. (2017). Perceived emotion genuineness: normative ratings for popular facial expression stimuli and the development of perceived-as-genuine and perceived-as-fake sets. Behavior Research Methods, 49(4), 1539-1562.

Dibeklioglu, H., Salah, A., \& Gevers, T. (2015). Recognition of Genuine Smiles. IEEE Transactions on Multimedia, 17(3), 279-294.

Duchenne, G. (1862). Mécanisme de la Physionomie Humaine. Paris: Jules Renouard. Ekman, P. (1992). Telling lies: Clues to deceit in the marketplace. New York: Norton. Ekman, P. (1999). Basic Emotions. In T. Dalgleish \& M. Power (Eds.), Handbook of cognition and emotion (pp. 45-60). John Wiley \& Sons, Ltd.

Ekman, P. (2003). Darwin, Deception, and Facial Expression. Annals of the New York Academy of Sciences, 1000, 205-221.

Ekman, P., \& Friesen, W. (1969a). Nonverbal leakage and clues to deception. Psychiatry, 32(1), 88-106.

Ekman, P., \& Friesen, W. (1969b). The repertoire of nonverbal behavior: Categories, origins, usage, and coding. Semiotica, l(1). 
Ekman, P., \& Friesen, W. (1975). Unmasking the face: A guide to recognizing emotions from facial clues.

Ekman, P., \& Friesen, W. (1982a). Felt, false, and miserable smiles. Journal of Nonverbal Behavior, 6(4), 238-252.

Ekman, P., \& Friesen, W. (1982b). Measuring facial movement with the Facial Action Coding System. In P. Ekman (Ed.), Emotion in the human face (Second edition ed., pp. 178211). Cambridge University Press.

Ekman, P., Friesen, W., \& Davidson, R. (1988). The Duchenne Smile: Emotional Expression And Brain Physiology II. Journal of Personality and Social Psychology, 58(2), 342-353.

Ekman, P., Friesen, W., \& Hager, J. (2002). FACS Investigator's Guide. Salt Lake: Network Information Research Coorporation.

Ekman, P., Friesen, W., \& O’Sullivan, M. (1988). Smiles when lying. Journal of Personality and Social Psychology, 54(3), 414-420.

Ekman, P., \& Rosenberg, E. (2005). What the face reveals: Basic and Applied Studies of Spontaneous Expression Using the Facial Action Coding System (FACS) (Second Edition ed.). Oxford University Press.

Galati, D., Scherer, K., \& Ricci-Bitti, P. (1997). Voluntary facial expression of emotion: comparing congenitally blind with normally sighted encoders. Journal of personality and social psychology, 73(6), 1363-79.

Girard, J., Shandar, G., Liu, Z., Cohn, J., Yin, L., \& Morency, L. (2019). Reconsidering the Duchenne Smile: Indicator of Positive Emotion or Artifact of Smile Intensity? PsyArXiv.

Girardi, D., Lanubile, F., \& Novielli, N. (2017). Emotion detection using noninvasive low cost sensors. In Affective computing and intelligent interaction (pp. 125-130).

Gruebler, A., \& Suzuki, K. (2014). Design of a Wearable Device for Reading Positive Expressions from Facial EMG Signals. IEEE Transactions on Affective Computing, PP(99), 1-1.

Guo, H., Zhang, X., Liang, J., \& Yan, W. (2018). The Dynamic Features of Lip Corners in Genuine and Posed Smiles. Frontiers in psychology, 9(February), 1-11. 
Henrich, J., Heine, S., \& Norenzayan, A. (2010). Most people are not WEIRD. Nature, 466(7302), 29-29.

Hoque, M., Morency, L., \& Picard, R. (2011). Are you friendly or just polite? Analysis of smiles in spontaneous face-to-face interactions. In S. D'Mello (Ed.), Affective computing and intelligent interaction. lecture notes in computer science (Vol. 6974, pp. 135-144). Springer Berlin Heidelberg.

Hourihan, K., Benjamin, A., \& Liu, X. (2012). A cross-race effect in metamemory:

Predictions of face recognition are more accurate for members of our own race. Journal of applied research in memory and cognition, 1(3), 158-162.

Iravantchi, Y., Zhang, Y., Bernitsas, E., Goel, M., \& Harrison, C. (2019). Interferi: Gesture Sensing using On-Body Acoustic Interferometry. In Conference on human factors in computing systems - proceedings. Association for Computing Machinery.

Jack, R., Garrod, O., \& Schyns, P. (2014). Dynamic Facial Expressions of Emotion Transmit an Evolving Hierarchy of Signals over Time. Current Biology, 24(2), $187-192$.

James, W. (1884). What is an emotion? Mind, os- $I X(34), 188-205$.

Janssen, J., Tacken, P., de Vries, J., van den Broek, E., Westerink, J., Haselager, P., \& IJsselsteijn, W. (2013). Machines outperform laypersons in recognizing emotions elicited by autobiographical recollection. Human-Computer Interaction, 28(6), 479517.

Jarlier, S., Grandjean, D., Delplanque, S., N’Diaye, K., Cayeux, I., Velazco, M. I., ... Scherer, K. (2011). Thermal analysis of facial muscles contractions. IEEE Transactions on Affective Computing, 2(1), 2-9.

Khan, M., Ward, R., \& Ingleby, M. (2009). Classifying pretended and evoked facial expressions of positive and negative affective states using infrared measurement of skin temperature. ACM Transactions on Applied Perception, 6(1).

Kreibig, S. (2010). Autonomic nervous system activity in emotion: a review. Biological Psychology, 84(3), 394-421. 
Krumhuber, E., \& Manstead, A. (2009). Can Duchenne smiles be feigned? New evidence on felt and false smiles. Emotion, 9(6), 807-820.

Krumhuber, E., \& Manstead, A. (2013). Effects of Dynamic Aspects of Facial Expressions : A Review. Emotion Review, 5(1), 41-46.

Mai, X., Ge, Y., Tao, L., Tang, H., Liu, C., \& Luo, Y. (2011). Eyes Are Windows to the Chinese Soul: Evidence from the Detection of Real and Fake Smiles. PLoS ONE, 6(5), e19903.

Masai, K., Kunze, K., Sugiura, Y., Ogata, M., Inami, M., \& Sugimoto, M. (2017).

Evaluation of facial expression recognition by a smart eyewear for facial direction changes, repeatability, and positional drift. ACM Transactions on Interactive Intelligent Systems, 7(4).

Masai, K., Sugiura, Y., Suzuki, K., Shimamura, S., Kunze, K., Ogata, M., Sugimoto, M. (2015). AffectiveWear: Towards Recognizing Affect in Real Life. Adjunct Proceedings of the 2015 ACM International Joint Conference on Pervasive and Ubiquitous Computing and Proceedings of the 2015 ACM International Symposium on Wearable Computers, 357-360.

Mavadati, M., Sanger, P., Mahoor, M., \& Street, S. (2016). Extended DISFA Dataset: Investigating Posed and Spontaneous Facial Expressions.

McDuff, D., Girard, J., \& el Kaliouby, R. (2017). Large-Scale Observational Evidence of Cross-Cultural Differences in Facial Behavior. Journal of Nonverbal Behavior, 41(1).

McGettigan, C., Walsh, E., Jessop, R., Agnew, Z., Sauter, D., Warren, J., \& Scott, S. (2015). Individual Differences in Laughter Perception Reveal Roles for Mentalizing and Sensorimotor Systems in the Evaluation of Emotional Authenticity. Cerebral Cortex, 25(1), 246-257.

Mehu, M., Mortillaro, M., Bänziger, T., \& Scherer, K. (2012). Reliable Facial Muscle Activation Enhances Recognizability and Credibility of Emotional Expression. Association, 12(4), 701-715.

Mehu, M., \& Scherer, K. (2012). A psycho-ethological approach to social signal processing. Cognitive Processing, 13(S2), 397-414. 
Meissner, C., \& Brigham, J. (2001). Thirty Years of Investigating the Own-Race Bias in Memory for Faces: A Meta-Analytic Review. Psychology, Public Policy, and Law, $7(1), 3-35$.

Messinger, D. (2002). Positive and negative: Infant facial expressions and emotions. Current Directions in Psychological Science, 11(1), 1-6.

Mignault, A., \& Chaudhuri, A. (2003). The many faces of a neutral face: Head tilt and perception of dominance and emotion. Journal of Nonverbal Behavior, 27(2),

$111-132$.

Nakayama, Y., Takano, Y., Matsubara, M., Suzuki, K., \& Terasawa, H. (2017). The sound of smile: Auditory biofeedback of facial EMG activity. Displays, 47, 32-39.

Namba, S., Kabir, R., Miyatani, M., \& Nakao, T. (2018). Dynamic Displays Enhance the Ability to Discriminate Genuine and Posed Facial Expressions of Emotion. Frontiers in Psychology, 9, 672.

Namba, S., Makihara, S., Kabir, R., Miyatani, M., \& Nakao, T. (2016). Spontaneous Facial Expressions Are Different from Posed Facial Expressions: Morphological Properties and Dynamic Sequences.

Nguyen, H. (2015). Estimation of Human Emotion by Analyzing of Visible Expressions and Thermal Facial Images (Unpublished doctoral dissertation). Japan Advanced Institute of Science and Technology.

Niedenthal, P., Rychlowska, M., Wood, A., \& Zhao, F. (2018). Heterogeneity of long-history migration predicts smiling, laughter and positive emotion across the globe and within the United States. PLOS ONE, 13(8), e0197651.

Oberman, L., Winkielman, P., \& Ramachandran, V. (2007). Face to face: blocking facial mimicry can selectively impair recognition of emotional expressions. Social neuroscience, 2(3-4), 167-78.

Oberman, L., Winkielman, P., \& Ramachandran, V. (2009). Slow echo : facial EMG evidence for the delay of spontaneous, but not voluntary, emotional mimicry in children with autism spectrum disorders. Developmental Science, 4, 510-520.

Oh, Y., See, J., LeNgo, A., Phan, R., \& Baskaran, V. (2018). A survey of automatic facial micro-expression analysis: Databases, methods, and challenges (Vol. 9). 
Frontiers Media S.A.

Orlowska, A., Krumhuber, E., Rychlowska, M., \& Szarota, P. (2018). Dynamics Matter:

Recognition of Reward, Affiliative, and Dominance Smiles From Dynamic vs. Static Displays. Frontiers in Psychology, 9, 938.

Perusquía-Hernández, M., Ayabe-Kanamura, S., \& Suzuki, K. (2019a). Human perception and biosignal-based identification of posed and spontaneous smiles.

PLOS ONE, 14(12), e0226328.

Perusquía-Hernández, M., Ayabe-Kanamura, S., \& Suzuki, K. (2019b). Posed and spontaneous smile assessment with wearable skin conductance measured from the neck and head movement. In (pp. 199-205).

Perusquía-Hernández, M., Ayabe-Kanamura, S., Suzuki, K., \& Kumano, S. (2019). The Invisible Potential of Facial Electromyography: A Comparison of EMG and Computer Vision when Distinguishing Posed from Spontaneous Smiles. In Proceedings of the 2019 chi conference on human factors in computing systems chi '19 (pp. 1-9). New York, New York, USA: ACM Press.

Perusquía-Hernández, M., Hirokawa, M., \& Suzuki, K. (2017a). Spontaneous and posed smile recognition based on spatial and temporal patterns of facial EMG. In Affective computing and intelligent interaction (pp. 537-541).

Perusquía-Hernández, M., Hirokawa, M., \& Suzuki, K. (2017b). A wearable device for fast and subtle spontaneous smile recognition. IEEE Transactions on Affective Computing, $8(4), 522-533$.

Rychlowska, M., Jack, R., Garrod, O., Schyns, P., Martin, J., \& Niedenthal, P. (2017).

Functional Smiles: Tools for Love, Sympathy, and War. Psychological Science, 28(9), $1259-1270$.

Rychlowska, M., Manstead, A., \& van der Schalk, J. (2019). The Many Faces of Smiles. In The social nature of emotion expression (pp. 227-245). Cham: Springer International Publishing.

Saha, P., Bhattacharjee, D., De, B., \& Nasipuri, M. (2016). Characterization and recognition of mixed emotional expressions in thermal face image. In Infrared imaging systems: Design, analysis, modeling, and testing xxvii (Vol. 9820, 
p. 98200S). SPIE.

Saito, C., Masai, K., \& Sugimoto, M. (2020). Classification of Spontaneous and Posed Smiles by Photo-reflective Sensors Embedded with Smart Eyewear. In Acm proceedings of the tangible and embodied interaction conference 2020.

Scherer, K., Schorr, A., \& Johnstone, T. (2001). Appraisal processes in emotion: theory, methods, research. Oxford University Press.

Schmidt, K., Ambadar, Z., Cohn, J., \& Reed, L. (2006). Movement differences between deliberate and spontaneous facial expressions: Zygomaticus major action in smiling. Journal of Nonverbal Behavior, 30(1), 37-52.

Schmidt, K., Bhattacharya, S., \& Denlinger, R. (2009). Comparison of deliberate and spontaneous facial movement in smiles and eyebrow raises. Nonverbal Behaviour, $33(1), 35-45$.

Schmidt, K., \& Cohn, J. (2001). Dynamics of facial expression: Normative characteristics and individual differences. In Ieee proceedings of international conference on multimedia and expo. (pp. 728-731). Tokyo: IEEE.

Silveira, F., Eriksson, B., Sheth, A., \& Sheppard, A. (2013). Predicting audience responses to movie content from electro-dermal activity signals. In Proceedings of the $2013 \mathrm{acm}$ international joint conference on pervasive and ubiquitous computing - ubicomp '13 ( $\mathrm{p}$. 707). New York, New York, USA: ACM Press.

Stewart, P., Bucy, E., \& Mehu, M. (2015). Strengthening bonds and connecting with followers. Politics and the Life Sciences, 34(1), 73-92.

Tassinary, L., \& Cacioppo, J. (1992). Unobservable Facial Actions and Emotion. Psychological Science, 3(1), 28-33.

Thibault, P., Gosselin, P., Brunel, M., \& Hess, U. (2009). Children's and adolescents' perception of the authenticity of smiles. Journal of Experimental Child Psychology, 102(3), 360-367.

Thibault, P., Levesque, M., Gosselin, P., \& Hess, U. (2012). The duchenne marker is not a universal signal of smile authenticity - but it can be learned! Social Psychology, 43(4), $215-221$. 
Valstar, M., Gunes, H., \& Pantic, M. (2007). How to distinguish posed from spontaneous smiles using geometric features. International Conf. Multimodal Interfaces, terfaces, 38-45.

van Boxtel, A. (2010). Facial EMG as a Tool for Inferring Affective States. In A. Spink, F. Grieco, O. Krips, L. Loijens, L. Noldus, \& P. Zimmerman (Eds.), Proceedings of measuring behavior (pp. 104-108). Eindhoven.

Vinciarelli, A., Pantic, M., \& Bourlard, H. (2009). Social signal processing: survey of an emerging domain. Image and Vision Computing, 27(12), 1743-1759.

Wang, S., Liu, Z., Wang, Z., Wu, G., Shen, P., He, S., \& Wang, X. (2013). Analyses of a multimodal spontaneous facial expression database. IEEE Transactions on Affective Computing, 4(1), 34-46.

Wang, S., Wu, C., \& Ji, Q. (2016). Capturing global spatial patterns for distinguishing posed and spontaneous expressions. Computer Vision and Image Understanding, 147, 69-76.

Wilson, M. (2002). Six views of embodied cognition. Psychonomic bulletin \& review, $9(4), 625-636$.

Yan, W., Qi, W., Jing, L., Chen, Y., \& Fu, X. (2013). How Fast are the Leaked Facial Expressions : The Duration of Micro-Expressions. Journal of Nonverbal Behavior, $37(4), 217-230$.

Yang, J., \& Wang, S. (2017). Capturing spatial and temporal patterns for distinguishing between posed and spontaneous expressions. In Proceedings of the 2017 acm on multimedia conference - mm '17 (pp. 469-477). New York, New York, USA: ACM Press.

Zhao, G., Huang, X., Taini, M., Li, S., \& Pietikäinen, M. (2011). Facial expression recognition from near-infrared videos. Image and Vision Computing, 29(9), 607619.

Zloteanu, M., Krumhuber, E., \& Richardson, D. (2018). Detecting Genuine and Deliberate Displays of Surprise in Static and Dynamic Faces. Frontiers in Psychology, 9, 1184. 